\title{
Existence results for double phase implicit obstacle problems involving multivalued operators
}

\author{
Shengda Zeng ${ }^{1,2} \cdot$ Yunru Bai ${ }^{2} \cdot$ Leszek Gasiński ${ }^{3} \cdot$ Patrick Winkert $^{4}$
}

Received: 16 March 2020 / Accepted: 31 July 2020 / Published online: 17 September 2020

(c) The Author(s) 2020

\begin{abstract}
In this paper we study implicit obstacle problems driven by a nonhomogenous differential operator, called double phase operator, and a multivalued term which is described by Clarke's generalized gradient. Based on a surjectivity theorem for multivalued mappings, Kluge's fixed point principle and tools from nonsmooth analysis, we prove the existence of at least one solution.
\end{abstract}

Mathematics Subject Classification $35 \mathrm{~J} 20 \cdot 35 \mathrm{~J} 25 \cdot 35 \mathrm{~J} 60$

\section{Introduction}

Given a bounded domain $\Omega$ in $\mathbb{R}^{N}, N \geq 2$, with Lipschitz boundary $\partial \Omega$, we study a double phase implicit obstacle problem with a multivalued operator given in the form

Communicated by P. Rabinowitz.

\author{
Shengda Zeng \\ zengshengda@163.com \\ Yunru Bai \\ yunrubai@163.com \\ Leszek Gasiński \\ leszek.gasinski@up.krakow.pl \\ Patrick Winkert \\ winkert@math.tu-berlin.de
}

1 Guangxi Colleges and Universities Key Laboratory of Complex System Optimization and Big Data Processing, Yulin Normal University, Yulin 537000, Guangxi, People's Republic of China

2 Faculty of Mathematics and Computer Science, Jagiellonian University in Krakow, ul. Lojasiewicza 6, 30-348 Kraków, Poland

3 Department of Mathematics, Pedagogical University of Cracow, Podchorazych 2, 30-084 Kraków, Poland

4 Institut für Mathematik, Technische Universität Berlin, Straße des 17. Juni 136, 10623 Berlin, Germany 


$$
\begin{aligned}
-\operatorname{div}\left(|\nabla u|^{p-2} \nabla u+\mu(x)|\nabla u|^{q-2} \nabla u\right)+\partial j(x, u) & \ni f(x) & & \text { in } \Omega, \\
u & =0 & & \text { on } \partial \Omega, \\
T(u) & \leq U(u), & &
\end{aligned}
$$

where $1<p<q<N, \mu: \bar{\Omega} \rightarrow[0, \infty)$ and $T, U: W_{0}^{1, \mathcal{H}}(\Omega) \rightarrow \mathbb{R}$ are given functions satisfying appropriate conditions (see Sect. 2). Here $W_{0}^{1, \mathcal{H}}(\Omega)$ is a subspace of the SobolevMusielak-Orlicz space $W^{1, \mathcal{H}}(\Omega)$ and $j: \Omega \times \mathbb{R} \rightarrow \mathbb{R}$ is supposed to be locally Lipschitz with respect to the second variable.

In this paper we prove the existence of at least one weak solution (see Definition (3.4)) of problem (1.1) by applying a surjectivity theorem for multivalued mappings, Kluge's fixed point principle and tools from nonsmooth analysis. In general, problem (1.1) combines several interesting phenomena like a double phase operator along with a multivalued mapping in form of Clarke's generalized gradient and an implicit obstacle given by the functions $T: W_{0}^{1, \mathcal{H}}(\Omega) \rightarrow \mathbb{R}$ and $U: W_{0}^{1, \mathcal{H}}(\Omega) \rightarrow(0,+\infty)$, see $\mathrm{H}(T)$ and $\mathrm{H}(U)$ in Sect. 3 for the precise conditions. Indeed, a solution $u \in W_{0}^{1, \mathcal{H}}(\Omega)$ of (1.1) has to belong to $K(u)$ which is the image of the multivalued map $K: W_{0}^{1, \mathcal{H}}(\Omega) \rightarrow 2^{W_{0}^{1, \mathcal{H}}(\Omega)}$ defined by

$$
K(u):=\left\{v \in W_{0}^{1, \mathcal{H}}(\Omega) \mid T(v)-U(u) \leq 0\right\} .
$$

To the best of our knowledge, this is the first work which combines a double phase phenomena along with Clarke's generalized gradient and an implicit obstacle. A main tool in our treatment will be a surjectivity result of Le [24] for multivalued mappings generated by the sum of a maximal monotone multivalued operator and a bounded multivalued pseudomonotone mapping.

One difficulty in the study of (1.1) is the occurrence of the so-called double phase operator defined by

$$
-\operatorname{div}\left(|\nabla u|^{p-2} \nabla u+\mu(x)|\nabla u|^{q-2} \nabla u\right), \quad u \in W_{0}^{1, \mathcal{H}}(\Omega),
$$

where $1<p<q<N$. Although this operator looks like the $(p, q)$-differential operator the difference is the weight function $\mu: \bar{\Omega} \rightarrow[0, \infty)$ which can have values in zero. That means we cannot search for weak solutions in the usual Sobolev space $W_{0}^{1, p}(\Omega)$, we need a certain type of a Sobolev-Musielak-Orlicz space equipped with the Luxemburg norm, see Sect. 2 for its definition. The idea to treat problems driven by the double phase operators goes back to the 1980s and the work of Zhikov [36] who introduced such classes of operators to describe models of strongly anisotropic materials by treating the functional

$$
\omega \mapsto \int\left(|\nabla \omega|^{p}+\mu(x)|\nabla \omega|^{q}\right) d x,
$$

see also Zhikov [37,38] and the monograph of Zhikov-Kozlov-Oleinik [39]. Integral functionals of the form (1.2) have been considered by several authors concerning regularity results and non-standard growth, see for example, Baroni-Colombo-Mingione [4,5,7], Baroni-Kussi-Mingione [6], Cupini-Marcellini-Mascolo [16], Colombo-Mingione [14,15], Marcellini [27,28] and the references therein.

In the case of single-valued equations in the whole space we refer to the works of Colasuonno-Squassina [13], Gasiński-Papageorgiou [17, Proposition 3.4], GasińskiWinkert [20,21], Liu-Dai [26], Perera-Squassina [33] concerning existence and multiplicity results. 
Works which are closely related to our paper dealing with certain types of double phase problems or multivalued problems can be found in Bahrouni-Rădulescu-Repovš [1], Bahrouni-Rădulescu-Winkert [2,3], Carl-Le-Motreanu [9], Cencelj-Rădulescu-Repovš [10], Clarke [12], Gasiński-Papageorgiou [18,19], Papageorgiou-Rădulescu-Repovš [30, 31], Rădulescu [34], Zhang-Rădulescu [35] and the references therein.

The paper is organized as follows. In Sect. 2 we recall the definition of the used function spaces, some embedding results and we state the surjectivity results of Le [24] for multivalued mappings as well as Kluge's fixed point theorem. In Sect. 3 we present the full assumptions on the data of problem (1.1), give the definition of the weak solution and consider an auxiliary problem defined in (3.7). Next, we prove some properties of the solution set of (3.7) stated as Theorem 3.6 whose proof is mainly based on tools from nonsmooth analysis in terms of multivalued mappings. Taking these results into account we are able to prove our main result which says that the solution set of (1.1) is nonempty, bounded and weakly closed in $W_{0}^{1, \mathcal{H}}(\Omega)$, see Theorem 3.5.

\section{Preliminaries}

In the whole paper we suppose that $\Omega$ is a bounded domain in $\mathbb{R}^{N}$. Given $1 \leq r \leq \infty, L^{r}(\Omega)$ and $L^{r}\left(\Omega ; \mathbb{R}^{N}\right)$ stand for the usual Lebesgue spaces equipped with the norm $\|\cdot\|_{p}$ while $W^{1, r}(\Omega)$ and $W_{0}^{1, r}(\Omega)$ denote the Sobolev spaces endowed with the norms $\|\cdot\|_{1, r}$ and $\|\cdot\|_{1, r, 0}$, respectively. By $r^{\prime}$, we denote the conjugate of $r \in(1, \infty)$, that is, $\frac{1}{r}+\frac{1}{r^{\prime}}=1$.

For the weight function in (1.1) we suppose the following condition:

$\mathrm{H}(\mu): \mu: \bar{\Omega} \rightarrow[0, \infty)$ is Lipschitz continuous and $1<p<q<N$ are chosen such that

$$
\frac{q}{p}<1+\frac{1}{N}
$$

Set $\mathbb{R}_{+}:=[0, \infty)$ and consider the modular function $\mathcal{H}: \Omega \times \mathbb{R}_{+} \rightarrow \mathbb{R}_{+}$given by

$$
\mathcal{H}(x, t)=t^{p}+\mu(x) t^{q} \quad \text { for all }(x, t) \in \Omega \times \mathbb{R}_{+} .
$$

The Musielak-Orlicz space $L^{\mathcal{H}}(\Omega)$ is defined by

$$
L^{\mathcal{H}}(\Omega)=\left\{u \mid u: \Omega \rightarrow \mathbb{R} \text { is measurable and } \rho_{\mathcal{H}}(u):=\int_{\Omega} \mathcal{H}(x,|u|) d x<+\infty\right\}
$$

equipped with the Luxemburg norm

$$
\|u\|_{\mathcal{H}}=\inf \left\{\tau>0 \mid \rho_{\mathcal{H}}\left(\frac{u}{\tau}\right) \leq 1\right\} .
$$

The space $L^{\mathcal{H}}(\Omega)$ is uniformly convex and so a reflexive Banach space. Furthermore, we introduce the seminormed function space $L_{\mu}^{q}(\Omega)$

$$
L_{\mu}^{q}(\Omega)=\left\{u \mid u: \Omega \rightarrow \mathbb{R} \text { is measurable and } \int_{\Omega} \mu(x)|u|^{q} d x<+\infty\right\}
$$

endowed with the seminorm

$$
\|u\|_{q, \mu}=\left(\int_{\Omega} \mu(x)|u|^{q} d x\right)^{\frac{1}{q}} .
$$


From Colasuonno-Squassina [13, Proposition 2.15 (i), (iv) and (v)] we know that the embeddings

$$
L^{q}(\Omega) \hookrightarrow L^{\mathcal{H}}(\Omega) \hookrightarrow L^{p}(\Omega) \cap L_{\mu}^{q}(\Omega)
$$

are continuous and so, by a simple calculation, we have

$$
\min \left\{\|u\|_{\mathcal{H}}^{p},\|u\|_{\mathcal{H}}^{q}\right\} \leq\|u\|_{p}^{p}+\|u\|_{q, \mu}^{q} \leq \max \left\{\|u\|_{\mathcal{H}}^{p},\|u\|_{\mathcal{H}}^{q}\right\}
$$

for all $u \in L^{\mathcal{H}}(\Omega)$.

The corresponding Sobolev-Musielak-Orlicz space $W^{1, \mathcal{H}}(\Omega)$ is defined by

$$
W^{1, \mathcal{H}}(\Omega)=\left\{u \in L^{\mathcal{H}}(\Omega):|\nabla u| \in L^{\mathcal{H}}(\Omega)\right\}
$$

and is equipped with the norm

$$
\|u\|_{1, \mathcal{H}}=\|\nabla u\|_{\mathcal{H}}+\|u\|_{\mathcal{H}},
$$

where $\|\nabla u\|_{\mathcal{H}}=\|\mid \nabla u\|_{\mathcal{H}}$.

The Sobolev-Musielak-Orlicz space with zero traces, denoted by $W_{0}^{1, \mathcal{H}}(\Omega)$, is the completion of $C_{0}^{\infty}(\Omega)$ in $W^{1, \mathcal{H}}(\Omega)$, that is,

$$
W_{0}^{1, \mathcal{H}}(\Omega)={\overline{C_{0}^{\infty}(\Omega)}}^{W^{1, \mathcal{H}}(\Omega)} .
$$

From the assumption on $\mu: \bar{\Omega} \rightarrow \mathbb{R}_{+}$in $\mathrm{H}(\mu)$ combined with Colasuonno-Squassina [13, Proposition 2.18], it is known that

$$
\|u\|_{1, \mathcal{H}, 0}=\|\nabla u\|_{\mathcal{H}} \text { for all } u \in W_{0}^{1, \mathcal{H}}(\Omega),
$$

defines an equivalent norm on $W_{0}^{1, \mathcal{H}}(\Omega)$. Based on this we obtain directly from (2.1) that

$$
\min \left\{\|u\|_{1, \mathcal{H}, 0}^{p},\|u\|_{1, \mathcal{H}, 0}^{q}\right\} \leq\|\nabla u\|_{p}^{p}+\|\nabla u\|_{q, \mu}^{q} \leq \max \left\{\|u\|_{1, \mathcal{H}, 0}^{p},\|u\|_{1, \mathcal{H}, 0}^{q}\right\}
$$

is true for all $u \in W_{0}^{1, \mathcal{H}}(\Omega)$. Moreover, both spaces $W^{1, \mathcal{H}}(\Omega)$ and $W_{0}^{1, \mathcal{H}}(\Omega)$ are uniformly convex and so reflexive Banach spaces as well.

From Colasuonno-Squassina [13, Proposition 2.15] we know that the embedding

$$
W_{0}^{1, \mathcal{H}}(\Omega) \hookrightarrow L^{r}(\Omega)
$$

is compact for each $1<r<p^{*}$, where $p^{*}$ stands for the critical exponent to $p$ given by

$$
p^{*}:=\frac{N p}{N-p} .
$$

Let us recall some properties of the eigenvalue problem for the $r$-Laplacian $(1<r<\infty)$ with homogeneous Dirichlet boundary condition given by

$$
\begin{aligned}
-\Delta_{r} u & =\lambda|u|^{r-2} u & & \text { in } \Omega, \\
u & =0 & & \text { on } \partial \Omega .
\end{aligned}
$$

It is known that the set $\sigma_{r}$ has a smallest element $\lambda_{1, r}$ which is positive, isolated, simple and it can be variationally characterized through

$$
\lambda_{1, r}=\inf \left\{\frac{\|\nabla u\|_{r}^{r}}{\|u\|_{r}^{r}}: u \in W_{0}^{1, r}(\Omega), u \neq 0\right\},
$$

see Lê [25]. 
Let $A: W_{0}^{1, \mathcal{H}}(\Omega) \rightarrow W_{0}^{1, \mathcal{H}}(\Omega)^{*}$ be the operator defined by

$$
\langle A(u), v\rangle_{\mathcal{H}}:=\int_{\Omega}\left(|\nabla u|^{p-2} \nabla u+\mu(x)|\nabla u|^{q-2} \nabla u\right) \cdot \nabla v d x,
$$

for $u, v \in W_{0}^{1, \mathcal{H}}(\Omega)$, where $\langle\cdot, \cdot\rangle_{\mathcal{H}}$ stands for the duality pairing between $W_{0}^{1, \mathcal{H}}(\Omega)$ and its dual space $W_{0}^{1, \mathcal{H}}(\Omega)^{*}$. The properties of the operator $A: W_{0}^{1, \mathcal{H}}(\Omega) \rightarrow W_{0}^{1, \mathcal{H}}(\Omega)^{*}$ can be summarized as follows, see Liu-Dai [26].

Proposition 2.1 The operator A defined by (2.5) is bounded, continuous, monotone (hence maximal monotone) and of type $\left(S_{+}\right)$.

The notion of pseudomonotonicity for multivalued operators is recalled in the next definition.

Definition 2.2 Let $X$ be a real reflexive Banach space. The operator $A: X \rightarrow 2^{X^{*}}$ is called pseudomonotone if the following conditions hold:

(i) the set $A(u)$ is nonempty, bounded, closed and convex for all $u \in X$;

(ii) $A$ is upper semicontinuous from each finite-dimensional subspace of $X$ to the weak topology on $X^{*}$;

(iii) if $\left\{u_{n}\right\} \subset X$ with $u_{n} \rightarrow u$ in $X$ and if $u_{n}^{*} \in A\left(u_{n}\right)$ is such that

$$
\limsup _{n \rightarrow \infty}\left\langle u_{n}^{*}, u_{n}-u\right\rangle_{X^{*} \times X} \leq 0,
$$

then to each element $v \in X$, exists $u^{*}(v) \in A(u)$ with

$$
\left\langle u^{*}(v), u-v\right\rangle_{X^{*} \times X} \leq \liminf _{n \rightarrow \infty}\left\langle u_{n}^{*}, u_{n}-v\right\rangle_{X^{*} \times X} .
$$

Let $X$ be a real Banach space with its dual space $X^{*}$. A function $J: X \rightarrow \mathbb{R}$ is said to be locally Lipschitz at $u \in X$ if there exist a neighborhood $N(u)$ of $u$ and a constant $L_{u}>0$ such that

$$
|J(w)-J(v)| \leq L_{u}\|w-v\|_{X} \text { for all } w, v \in N(u) .
$$

Definition 2.3 Let $J: X \rightarrow \mathbb{R}$ be a locally Lipschitz function and let $u, v \in X$. The generalized directional derivative $J^{\circ}(u ; v)$ of $J$ at the point $u$ in the direction $v$ is defined by

$$
J^{\circ}(u ; v):=\limsup _{w \rightarrow u, t \downarrow 0} \frac{J(w+t v)-J(w)}{t} .
$$

The generalized gradient $\partial J: X \rightarrow 2^{X^{*}}$ of $J: X \rightarrow \mathbb{R}$ is defined by

$$
\partial J(u):=\left\{\xi \in X^{*} \mid J^{\circ}(u ; v) \geq\langle\xi, v\rangle_{X^{*} \times X} \text { for all } v \in X\right\} \text { for all } u \in X .
$$

The next proposition collects some basic results, see for example, Migórski-OchalSofonea [29, Proposition 3.23].

Proposition 2.4 Let $J: X \rightarrow \mathbb{R}$ be locally Lipschitz with Lipschitz constant $L_{u}>0$ at $u \in X$. Then we have the following:

(i) The function $v \mapsto J^{\circ}(u ; v)$ is positively homogeneous, subadditive, and satisfies

$$
\left|J^{\circ}(u ; v)\right| \leq L_{u}\|v\|_{X} \text { for all } v \in X .
$$

(ii) The function $(u, v) \mapsto J^{\circ}(u ; v)$ is upper semicontinuous. 
(iii) For each $u \in X, \partial J(u)$ is a nonempty, convex, and weak* compact subset of $X^{*}$ with $\|\xi\|_{X^{*}} \leq L_{u}$ for all $\xi \in \partial J(u)$.

(iv) $J^{\circ}(u ; v)=\max \left\{\langle\xi, v\rangle_{X^{*} \times X} \mid \xi \in \partial J(u)\right\}$ for all $v \in X$.

(v) The multivalued function $X \ni u \mapsto \partial J(u) \subset X^{*}$ is upper semicontinuous from $X$ into $w^{*}-X^{*}$.

Since our results are based on fixed point results, so we now recall the fixed point theorem of Kluge [23].

Theorem 2.5 Let $Z$ be a real reflexive Banach space and let $C \subset Z$ be nonempty, closed and convex. Assume that $\Psi: C \rightarrow 2^{C}$ is a multivalued mapping such that for every $u \in C$, the set $\Psi(u)$ is nonempty, closed, and convex and the graph of $\Psi$ is sequentially weakly closed. If either $C$ is bounded or $\Psi(C)$ is bounded, then the map $\Psi$ has at least one fixed point in $C$.

Finally, we end this section by recalling the following surjectivity theorem for multivalued mappings which was proved by Le [24, Theorem 2.2]. We use the notation $B_{R}(0):=\{u \in$ $\left.X:\|u\|_{X}<R\right\}$.

Theorem 2.6 Let $X$ be a real reflexive Banach space, let $F: D(F) \subset X \rightarrow 2^{X^{*}}$ be a maximal monotone operator, let $G: D(G)=X \rightarrow 2^{X^{*}}$ be a bounded multivalued pseudomonotone operator and let $L \in X^{*}$. Assume that there exist $u_{0} \in X$ and $R \geq\left\|u_{0}\right\|_{X}$ such that $D(F) \cap B_{R}(0) \neq \varnothing$ and

$$
\left\langle\xi+\eta-L, u-u_{0}\right\rangle_{X^{*} \times X}>0
$$

for all $u \in D(F)$ with $\|u\|_{X}=R$, for all $\xi \in F(u)$ and for all $\eta \in G(u)$. Then the inclusion

$$
F(u)+G(u) \ni L
$$

has a solution in $D(F)$.

\section{Main results}

We impose the following assumptions for the data of problem (1.1).

$\mathrm{H}(f): f \in L^{p^{\prime}}(\Omega)$.

$\mathrm{H}(j): j: \Omega \times \mathbb{R} \rightarrow \mathbb{R}$ is such that

(i) $x \mapsto j(x, s)$ is measurable for all $s \in \mathbb{R}$ and there exists a function $l \in L^{q_{1}}(\Omega)$ with $q_{1} \in\left(1, p^{*}\right)$ such that the function $x \mapsto j(x, l(x))$ belongs to $L^{1}(\Omega)$;

(ii) $s \mapsto j(x, s)$ is locally Lipschitz continuous for a. a. $x \in \Omega$.

(iii) there exist $\theta \geq 1$ with $\theta \leq \min \left\{q_{1}, p\right\}, \alpha_{j} \geq 0$ with $\alpha_{j}<\lambda_{1, p}^{-1} \delta_{\theta}$ and $\beta_{j} \in L_{+}^{1}(\Omega)$ such that

$$
j^{\circ}(x, s ;-s) \leq \alpha_{j}|s|^{\theta}+\beta_{j}(x)
$$

for a.a. $x \in \Omega$ and for all $s \in \mathbb{R}$, where $\delta_{\theta}$ is defined by

$$
\delta_{\theta}= \begin{cases}1 & \text { if } \theta=p, \\ +\infty & \text { otherwise, }\end{cases}
$$

and $\lambda_{1, p}$ denotes the first eigenvalue of the Dirichlet eigenvalue problem for the $p$-Laplacian, see (2.4); 
(iv) there exist $c_{j} \geq 0$ and $\gamma_{j} \in L_{+}^{\frac{q_{1}}{q_{1}-1}}(\Omega)$ satisfying

$$
|\xi| \leq c_{j}|r|^{q_{1}-1}+\gamma_{j}(x)
$$

for a. a. $x \in \Omega$, for all $\xi \in \partial j(x, s)$ and for all $s \in \mathbb{R}$, where $\partial j(x, s)$ stands for the generalized gradient of $j$ with respect to the variable $s$ and $q_{1}$ is given in (i);

(v) there exists a constant $m_{j} \geq 0$ such that

$$
\left(\xi_{1}-\xi_{2}\right)\left(s_{1}-s_{2}\right) \geq-m_{j}\left|s_{1}-s_{2}\right|^{p}
$$

for a.a. $x \in \Omega$ and for all $s_{1}, s_{2} \in \mathbb{R}$ whenever $\xi_{1} \in \partial j\left(x, s_{1}\right)$ and $\xi_{2} \in \partial j\left(x, s_{2}\right)$.

$\mathrm{H}(T): T: W_{0}^{1, \mathcal{H}}(\Omega) \rightarrow \mathbb{R}$ is positively homogeneous and subadditive such that

$$
T(u) \leq \limsup _{n \rightarrow \infty} T\left(u_{n}\right)
$$

whenever $\left\{u_{n}\right\} \subset W_{0}^{1, \mathcal{H}}(\Omega)$ is such that $u_{n} \rightarrow u$ in $W_{0}^{1, \mathcal{H}}(\Omega)$ for some $u \in W_{0}^{1, \mathcal{H}}(\Omega)$. $\mathrm{H}(U): U: W_{0}^{1, \mathcal{H}}(\Omega) \rightarrow(0,+\infty)$ is weakly continuous, that is, for any sequence $\left\{u_{n}\right\} \subset$ $W_{0}^{1, \mathcal{H}}(\Omega)$ such that $u_{n} \rightarrow u$ for some $u \in W_{0}^{1, \mathcal{H}}(\Omega)$, we have

$$
U\left(u_{n}\right) \rightarrow U(u)
$$

Remark 3.1 (a) Assumption $\mathrm{H}(j)$ (v) is usually called the relaxed monotone condition for the locally Lipschitz function $s \mapsto j(x, s)$, see for example, Migórski-Ochal-Sofonea [29]. It is equivalent to the inequality

$$
j^{\circ}\left(x, s_{1} ; s_{2}-s_{1}\right)+j^{\circ}\left(x, s_{2} ; s_{1}-s_{2}\right) \leq m_{j}\left|s_{1}-s_{2}\right|^{p}
$$

for a. a. $x \in \Omega$ and for all $s_{1}, s_{2} \in \mathbb{R}$.

(b) Positive homogeneity and subadditivity of $T$ imply that $T$ is also a convex function. On the other hand, it is not difficult to see that if $T: W_{0}^{1, \mathcal{H}}(\Omega) \rightarrow \mathbb{R}$ is lower semicontinuous, then inequality (3.1) holds automatically.

We introduce the following multivalued map $K: W_{0}^{1, \mathcal{H}}(\Omega) \rightarrow 2^{W_{0}^{1, \mathcal{H}}}(\Omega)$ defined by

$$
K(u):=\left\{v \in W_{0}^{1, \mathcal{H}}(\Omega) \mid T(v)-U(u) \leq 0\right\}
$$

for all $u \in W_{0}^{1, \mathcal{H}}(\Omega)$.

Lemma 3.2 Let $U: W_{0}^{1, \mathcal{H}}(\Omega) \rightarrow(0,+\infty)$ and $T: W_{0}^{1, \mathcal{H}}(\Omega) \rightarrow \mathbb{R}$ be such that $H(T)$ holds. Then, for each $u \in W_{0}^{1, \mathcal{H}}(\Omega)$, the set $K(u)$ is nonempty, closed and convex in $W_{0}^{1, \mathcal{H}}(\Omega)$.

Proof For any fixed $u \in W_{0}^{1, \mathcal{H}}(\Omega)$ it is clear that $U(u)>0$. Since $T$ is positively homogeneous, we have $T(0)=0$. This implies $T(0)=0<U(u)$, that is, $0 \in K(u)$ and so, $K(u)$ is nonempty.

Let $\left\{v_{n}\right\} \subset K(u)$ be a sequence such that $v_{n} \rightarrow v$ in $W_{0}^{1, \mathcal{H}}(\Omega)$ for some $v \in W_{0}^{1, \mathcal{H}}(\Omega)$. Hence, for each $n \in \mathbb{N}$, one has

$$
T\left(v_{n}\right) \leq U(u)
$$

Passing to the upper limit as $n \rightarrow \infty$ and taking inequality (3.1) into account, we obtain

$$
T(v) \leq \limsup _{n \rightarrow \infty} T\left(v_{n}\right) \leq U(u) .
$$


Hence, $v \in K(u)$ which shows that $K(u)$ is closed.

Let $v_{1}, v_{2} \in K(u)$ and $t \in(0,1)$ be arbitrary. We set $v_{t}=t v_{1}+(1-t) v_{2}$. Then, we have $T\left(v_{i}\right) \leq U(u)$ for $i=1,2$. By the convexity of $T$, see Remark 3.1, it follows that

$$
T\left(v_{t}\right) \leq t T\left(v_{1}\right)+(1-t) T\left(v_{2}\right) \leq t U(u)+(1-t) U(u)=U(u) .
$$

Thus $v_{t} \in K(u)$. Therefore, we conclude that $K(u)$ is a convex set in $W_{0}^{1, \mathcal{H}}(\Omega)$.

Let us introduce the functional $J: L^{q_{1}}(\Omega) \rightarrow \mathbb{R}$ defined by

$$
J(u):=\int_{\Omega} j(x, u(x)) d x \text { for all } u \in L^{q_{1}}(\Omega) .
$$

From hypotheses $\mathrm{H}(j)$ and the definition of $J$ in (3.3), the next lemma is a direct consequence of Migórski-Ochal-Sofonea [29, Theorem 3.47].

Lemma 3.3 Under the assumptions $\mathrm{H}(j)(\mathrm{i})$ - (iv), the following hold:

(i) $J: L^{q_{1}}(\Omega) \rightarrow \mathbb{R}$ is locally Lipschitz continuous;

(ii) there hold

$$
\begin{aligned}
J^{\circ}(u ; v) & \leq \int_{\Omega} j^{\circ}(x, u(x) ; v(x)) d x, \\
J^{\circ}(u ;-u) & \leq \alpha_{j}\|u\|_{\theta}^{\theta}+\left\|\beta_{j}\right\|_{1}
\end{aligned}
$$

for all $u, v \in L^{q_{1}}(\Omega)$;

(iii) for each $u \in L^{q_{1}}(\Omega)$, one has

$$
\begin{aligned}
& \partial J(u) \subset \int_{\Omega} \partial j(x, u(x)) d x \\
&\|\xi\|_{q_{1}^{\prime}} \leq c_{J}\left(1+\|u\|_{q_{1}}^{q_{1}-1}\right) \quad \text { for all } \xi \in \partial J(u)
\end{aligned}
$$

with some $c_{J}>0$.

Moreover, if condition $\mathrm{H}(j)(\mathrm{v})$ holds, then the inequality

$$
J^{\circ}(u ; v-u)+J^{\circ}(v ; u-v) \leq m_{j}\|u-v\|_{p}^{p}
$$

is satisfied for all $u, v \in W_{0}^{1, \mathcal{H}}(\Omega)$.

The weak solutions for problem (1.1) are understood in the following sense.

Definition 3.4 We say that $u \in W_{0}^{1, \mathcal{H}}(\Omega)$ is a weak solution of problem (1.1) if $u \in K(u)$ and

$$
\begin{aligned}
& \int_{\Omega}\left(|\nabla u|^{p-2} \nabla u \cdot \nabla(v-u)+\mu(x)|\nabla u|^{q-2} \nabla u \cdot \nabla(v-u)\right) d x \\
& \quad+\int_{\Omega} j^{\circ}(x, u ; v-u) d x \geq \int_{\Omega} f(x)(v-u) d x
\end{aligned}
$$

for all $v \in K(u)$, where the multivalued function $K$ is given by (3.2).

Our main results read as follows. 
Theorem 3.5 Assume that $\mathrm{H}(\mu), \mathrm{H}(f), \mathrm{H}(j), \mathrm{H}(T)$ and $\mathrm{H}(U)$ are satisfied. If $p \geq 2$ and the smallness condition

$$
m_{j} \lambda_{1, p}^{-1}<c(p)
$$

holds, then the set of solutions for problem (1.1), denoted by $\mathcal{S}$, is nonempty, bounded and weakly closed in $W_{0}^{1, \mathcal{H}}(\Omega)$, where $c(p)>0$ is the largest constant such that

$$
\left(|x|^{p-2} x-|y|^{p-2} y\right) \cdot(x-y) \geq c(p)|x-y|^{p} \text { for all } x, y \in \mathbb{R}^{N} .
$$

From Lemma 3.3(ii) we see that if $u \in W_{0}^{1, \mathcal{H}}(\Omega)$ solves the following problem:

Find $u \in W_{0}^{1, \mathcal{H}}(\Omega)$ such that $u \in K(u)$ and

$$
\begin{aligned}
& \int_{\Omega}\left(|\nabla u|^{p-2} \nabla u \cdot \nabla(v-u)+\mu(x)|\nabla u|^{q-2} \nabla u \cdot \nabla(v-u)\right) d x \\
& \quad+J^{\circ}(u ; v-u) \geq \int_{\Omega} f(x)(v-u) d x
\end{aligned}
$$

for all $v \in K(u)$, then $u$ is also a weak solution of problem (1.1). Based on this fact, in the sequel, we are going to explore the existence of solutions for problem (3.6).

To this end, we now introduce the following auxiliary problem:

For given $w \in W_{0}^{1, \mathcal{H}}(\Omega)$, find $u \in K(w)$ such that

$$
\begin{aligned}
& \int_{\Omega}\left(|\nabla u|^{p-2} \nabla u \cdot \nabla(v-u)+\mu(x)|\nabla u|^{q-2} \nabla u \cdot \nabla(v-u)\right) d x \\
& \quad+J^{\circ}(u ; v-u) \geq \int_{\Omega} f(x)(v-u) d x
\end{aligned}
$$

for all $v \in K(w)$. Setting the multivalued $\Gamma: W_{0}^{1, \mathcal{H}}(\Omega) \rightarrow 2^{W_{0}^{1, \mathcal{H}}(\Omega)}$ by

$$
\Gamma(w):=\left\{u \in W_{0}^{1, \mathcal{H}}(\Omega) \mid u \text { solves problem (3.7)corresponding to } w\right\}
$$

for all $w \in W_{0}^{1, \mathcal{H}}(\Omega)$, it is not difficult to verify that $u \in W_{0}^{1, \mathcal{H}}(\Omega)$ is a fixed point of $\Gamma$ if and only if $u$ solves problem (3.6). Motivated by this fact, we shall employ Kluge's fixed point principle, see Theorem 2.5, in order to show that $\Gamma$ has at least one fixed point in $W_{0}^{1, \mathcal{H}}(\Omega)$.

Theorem 3.6 Let $U: W_{0}^{1, \mathcal{H}}(\Omega) \rightarrow(0,+\infty)$. Under the assumptions $\mathrm{H}(\mu), H(f), H(j)(i)-(i v)$ and $\mathrm{H}(T)$, the following hold:

(i) for each $w \in W_{0}^{1, \mathcal{H}}(\Omega)$, the set of solutions to problem (3.7) is nonempty, bounded and closed in $W_{0}^{1, \mathcal{H}}(\Omega)$, that is, $\Gamma$ has nonempty, bounded and closed values.

(ii) if, in addition, $p \geq 2, \mathrm{H}(j)(\mathrm{v})$ and the smallness condition (3.5) are fulfilled, then for each $w \in W_{0}^{1, \mathcal{H}}(\Omega)$, problem (3.7) has a unique solution $u_{w} \in W_{0}^{1, \mathcal{H}}(\Omega)$, namely, $\Gamma(w)=\left\{u_{w}\right\}$.

Proof (i) For any fixed $w \in W_{0}^{1, \mathcal{H}}(\Omega)$, we consider the indicator function $I_{K(w)}: W_{0}^{1, \mathcal{H}}(\Omega) \rightarrow$ $\overline{\mathbb{R}}:=\mathbb{R} \cup\{+\infty\}$ of $K(w)$ defined by

$$
I_{K(w)}(u):= \begin{cases}0 & \text { if } u \in K(w) \\ +\infty & \text { otherwise }\end{cases}
$$


From the fact that $f \in L^{p^{\prime}}(\Omega) \subset W_{0}^{1, \mathcal{H}}(\Omega)^{*}$, problem (3.7) can be expressed as the variational-hemivariational inequality: Find $u \in W_{0}^{1, \mathcal{H}}(\Omega)$ such that

$$
\langle A u, v-u\rangle_{\mathcal{H}}+J^{\circ}(u ; v-u)+I_{K(w)}(v)-I_{K(w)}(u) \geq\langle f, v-u\rangle_{\mathcal{H}}
$$

for all $v \in W_{0}^{1, \mathcal{H}}(\Omega)$, where $A: W_{0}^{1, \mathcal{H}}(\Omega) \rightarrow W_{0}^{1, \mathcal{H}}(\Omega)^{*}$ is the double phase operator defined in (2.5). Employing the first separation theorem, see for example, Papageorgiou-Winkert [32, Theorem 3.1.57], it is not difficult to see that inequality problem (3.8) is equivalent to the following inclusion problem: Find $u \in W_{0}^{1, \mathcal{H}}(\Omega)$ such that

$$
A u+\partial J(u)+\partial_{C} I_{K(w)}(u) \ni f,
$$

where the notation $\partial_{C} I_{K(w)}$ stands for the subdifferential of $I_{K(w)}$ in the sense of convex analysis.

First, we are going to apply the surjectivity theorem for multivalued mappings, see Theorem 2.6, in order to prove that problem (3.9) has at least one solution in $W_{0}^{1, \mathcal{H}_{(}}(\Omega)$. In fact, we have the following claims.

Claim $1 A+\partial J: W_{0}^{1, \mathcal{H}}(\Omega) \rightarrow 2^{W_{0}^{1, \mathcal{H}}}(\Omega)^{*}$ is a bounded pseudomonotone multivalued operator such that for each $u \in W_{0}^{1, \mathcal{H}}(\Omega)$, the set $A(u)+\partial J(u)$ is closed and convex in $W_{0}^{1, \mathcal{H}}(\Omega)^{*}$.

Indeed, Proposition 2.4 and Lemma 3.3 imply that for each $u \in W_{0}^{1, \mathcal{H}}(\Omega)$, the set $A(u)+$ $\partial J(u)$ is closed and convex in $W_{0}^{1, \mathcal{H}}(\Omega)^{*}$. Besides, Proposition 2.1, Lemma 3.3(iii), (2.3) and the fact that $p_{1}<p^{*}$ guarantee that

$$
W_{0}^{1, \mathcal{H}}(\Omega) \ni u \mapsto A(u)+\partial J(u) \subset W_{0}^{1, \mathcal{H}}(\Omega)^{*} \text { is a bounded map. }
$$

Next are going to apply Proposition 3.8 in Migórski-Ochal-Sofonea [29] in order to conclude that $W_{0}^{1, \mathcal{H}}(\Omega) \ni u \mapsto A(u)+\partial J(u) \subset W_{0}^{1, \mathcal{H}}(\Omega)^{*}$ is upper semicontinuous from $W_{0}^{1, \mathcal{H}}(\Omega)$ to $W_{0}^{1, \mathcal{H}}(\Omega)^{*}$ equipped with the weak topology. It is sufficient to show that for any weakly closed subset $D$ in $W_{0}^{1, \mathcal{H}}(\Omega)^{*}$, the set $(A+\partial J)^{-}(D)$ is closed in $W_{0}^{1, \mathcal{H}}(\Omega)$.

Let $\left\{u_{n}\right\} \subset(A+\partial J)^{-}(D)$ be a sequence such that

$$
u_{n} \rightarrow u \text { in } W_{0}^{1, \mathcal{H}}(\Omega) \text { for some } u \in W_{0}^{1, \mathcal{H}}(\Omega) .
$$

Hence, for each $n \in \mathbb{N}$, we are able to find $\xi_{n} \in \partial J\left(u_{n}\right)$ such that

$$
u_{n}^{*}:=A u_{n}+\xi_{n} \in\left(A\left(u_{n}\right)+\partial J\left(u_{n}\right)\right) \cap D .
$$

The continuity of $A$, see Proposition 2.1, implies that $A\left(u_{n}\right) \rightarrow A(u)$ in $W_{0}^{1, \mathcal{H}}(\Omega)^{*}$. Lemma 3.3(iii) and convergence (3.10) imply that the sequence $\left\{\xi_{n}\right\}$ is bounded in $W_{0}^{1, \mathcal{H}}(\Omega)^{*}$. So, without any loss of generality, we may assume that

$$
\xi_{n} \rightarrow \xi \text { in } W_{0}^{1, \mathcal{H}}(\Omega)^{*} .
$$

Recall that $\partial J$ is upper semicontinuous from $W_{0}^{1, \mathcal{H}}(\Omega)$ to $W_{0}^{1, \mathcal{H}}(\Omega)^{*}$ equipped with the weak topology and has bounded, convex, closed values, see Proposition 2.4(iv). Hence, it has a closed graph in $W_{0}^{1, \mathcal{H}}(\Omega) \times W_{0}^{1, \mathcal{H}}(\Omega)^{*}$, see Kamenskii-Obukhovskii-Zecca [22, Theorem 1.1.4]. But, thanks to the weak closedness of $D$, we derive that $A(u)+\xi \in D$ and $\xi \in \partial J(u)$, which provides that $u \in(A+\partial J)^{-}(D)$. Consequently, $A+\partial J$ is upper semicontinuous from $W_{0}^{1, \mathcal{H}}(\Omega)$ to $W_{0}^{1, \mathcal{H}}(\Omega)^{*}$ equipped with the weak topology. 
We now prove that $A+\partial J$ is pseudomonotone. Let $\left\{u_{n}\right\}$ and $\left\{u_{n}^{*}\right\}$ be sequences such that

$$
\begin{aligned}
& u_{n} \rightarrow u \text { in } W_{0}^{1, \mathcal{H}}(\Omega), \\
& u_{n}^{*} \in A\left(u_{n}\right)+\partial J\left(u_{n}\right) \quad \text { with } \limsup _{n \rightarrow \infty}\left\langle u_{n}^{*}, u_{n}-u\right\rangle_{\mathcal{H}} \leq 0 .
\end{aligned}
$$

Our goal is to produce for each $v \in W_{0}^{1, \mathcal{H}}(\Omega)$ an element $u^{*}(v) \in A(u)+\partial J(u)$ such that

$$
\liminf _{n \rightarrow \infty}\left\langle u_{n}^{*}, u_{n}-v\right\rangle_{\mathcal{H}} \geq\left\langle u^{*}(v), u-v\right\rangle_{\mathcal{H}}
$$

From (3.12), there is a sequence $\left\{\xi_{n}\right\} \subset W_{0}^{1, \mathcal{H}}(\Omega)^{*}$ such that for each $n \in \mathbb{N}, \xi_{n} \in \partial J\left(u_{n}\right)$ and

$$
u_{n}^{*}=A\left(u_{n}\right)+\xi_{n}
$$

From (3.12), and the above equality it follows that

$$
\limsup _{n \rightarrow \infty}\left\langle A u_{n}, u_{n}-u\right\rangle_{\mathcal{H}}+\liminf _{n \rightarrow \infty}\left\langle\xi_{n}, u_{n}-u\right\rangle_{\mathcal{H}} \leq 0
$$

Applying (3.11) and the compact embedding of $W_{0}^{1, \mathcal{H}}(\Omega)$ into $L^{q_{1}}(\Omega)$, see (2.3), we have

$$
u_{n} \rightarrow u \text { in } L^{q_{1}}(\Omega)
$$

By virtue of Theorem 2.2 of Chang [11], we know that

$$
\partial\left(\left.J\right|_{W_{0}^{1, \mathcal{H}}(\Omega)}\right)(u) \subset \partial\left(\left.J\right|_{L^{q_{1}(\Omega)}}\right)(u) \text { for all } u \in W_{0}^{1, \mathcal{H}}(\Omega),
$$

which shows that

$$
\left\langle\xi_{n}, u_{n}-u\right\rangle_{\mathcal{H}}=\left\langle\xi_{n}, u_{n}-u\right\rangle_{L^{q_{1}}(\Omega)} .
$$

Additionally, Lemma 3.3(iii) and the boundedness of $\left\{u_{n}\right\}$ in $W_{0}^{1, \mathcal{H}}(\Omega)$ entail that the sequence $\left\{\xi_{n}\right\}$ is bounded both in $L^{q_{1}}(\Omega)$ and $W_{0}^{1, \mathcal{H}}(\Omega)^{*}$. Then, we pass to the limit in (3.15) as $n \rightarrow \infty$, to get

$$
\lim _{n \rightarrow \infty}\left\langle\xi_{n}, u_{n}-u\right\rangle_{\mathcal{H}}=\lim _{n \rightarrow \infty}\left\langle\xi_{n}, u_{n}-u\right\rangle_{L^{q_{1}(\Omega)}}=0 .
$$

Combining this with (3.14) leads to

$$
\limsup _{n \rightarrow \infty}\left\langle A u_{n}, u_{n}-u\right\rangle_{\mathcal{H}}=\limsup _{n \rightarrow \infty}\left\langle A u_{n}, u_{n}-u\right\rangle_{\mathcal{H}}+\liminf _{n \rightarrow \infty}\left\langle\xi_{n}, u_{n}-u\right\rangle_{\mathcal{H}} \leq 0 .
$$

Hence, since $A$ is of type ( $S_{+}$), see Proposition 2.1, and (3.11) yields $u_{n} \rightarrow u$ in $W_{0}^{1, \mathcal{H}}(\Omega)$. On the other hand, by the reflexivity of $W_{0}^{1, \mathcal{H}}(\Omega)^{*}$ and the boundedness of $\left\{\xi_{n}\right\} \subset W_{0}^{1, \mathcal{H}}(\Omega)^{*}$, we can suppose that

$$
\xi_{n} \rightarrow \xi \text { in } W_{0}^{1, \mathcal{H}}(\Omega)^{*} \text { for some } \xi \in W_{0}^{1, \mathcal{H}}(\Omega)^{*} .
$$

Then, from Kamenskii-Obukhovskii-Zecca [22, Theorem 1.1.4], we can conclude that $\xi \in$ $\partial J(u)$. Because of

$$
\liminf _{n \rightarrow \infty}\left\langle u_{n}^{*}, u_{n}-v\right\rangle_{\mathcal{H}}=\liminf _{n \rightarrow \infty}\left\langle A\left(u_{n}\right)+\xi_{n}, u_{n}-v\right\rangle_{\mathcal{H}}=\langle A(u)+\xi, u-v\rangle_{\mathcal{H}},
$$

it is clear that (3.13) holds with $u^{*}=A(u)+\xi \in A(u)+\partial J(u)$. We conclude that $A+\partial J$ is pseudomonotone. This proves Claim 1 . 
Claim 2 There exists $R>0$ such that

$$
\langle A u+\xi+\eta-f, u\rangle_{\mathcal{H}}>0
$$

for all $u \in K(w)$ with $\|u\|_{1, \mathcal{H}, 0}=R$, for all $\xi \in \partial J(u)$ and for all $\eta \in \partial_{C}\left(I_{K(w)}\right)(u)$.

Let $u \in W_{0}^{1, \mathcal{H}}(\Omega)$ be fixed. Since $0 \in K(w)$ and $f \in L^{p^{\prime}}(\Omega) \subset W_{0}^{1, \mathcal{H}}(\Omega)^{*}$, for any $\xi \in \partial J(u)$ and $\eta \in \partial_{C}\left(I_{K(w)}\right)(u)$, we can find

$$
\begin{aligned}
\langle A u & +\xi+\eta-f, u\rangle_{\mathcal{H}} \\
\geq & \int_{\Omega}|\nabla u|^{p-2} \nabla u \cdot \nabla u d x+\int_{\Omega} \mu(x)|\nabla u|^{q-2} \nabla u \cdot \nabla u d x \\
& +\int_{\Omega} \xi u d x+I_{K(w)}(u)-I_{K(w)}(0)-\|f\|_{W_{0}^{1, \mathcal{H}}(\Omega)^{*}}\|u\|_{1, \mathcal{H}, 0} \\
\geq & \|\nabla u\|_{p}^{p}+\|\nabla u\|_{q, \mu}^{q}-\int_{\Omega} \xi[-u] d x+I_{K(w)}(u)-\|f\|_{W_{0}^{1, \mathcal{H}}(\Omega)^{*}}\|u\|_{1, \mathcal{H}, 0} \\
\geq & \|\nabla u\|_{p}^{p}+\|\nabla u\|_{q, \mu}^{q}-J^{\circ}(u ;-u)+I_{K(w)}(u)-\|f\|_{W_{0}^{1, \mathcal{H}}(\Omega)^{*}}\|u\|_{1, \mathcal{H}, 0} .
\end{aligned}
$$

Note that $I_{K(w)}: W_{0}^{1, \mathcal{H}}(\Omega) \rightarrow \overline{\mathbb{R}}$ is a proper, convex and lower semicontinuous function. Hence, we can apply Proposition 1.10 of Brezis [8] to find $a_{K(w)}, b_{K(w)}>0$ such that

$$
I_{K(w)}(v) \geq-a_{K(w)}\|v\|_{1, \mathcal{H}, 0}-b_{K(w)} \text { for all } v \in W_{0}^{1, \mathcal{H}}(\Omega) .
$$

In addition, by Lemma 3.3(ii), we have

$$
J^{\circ}(u ;-u) \leq \alpha_{j}\|u\|_{\theta}^{\theta}+\left\|\beta_{j}\right\|_{1} .
$$

We consider now the two cases $\theta<p$ and $\theta=p$. Suppose first $\theta<p$ and let $c(\theta)>0$ be such that

$$
\|u\|_{\theta} \leq c(\theta)\|u\|_{1, \mathcal{H}, 0} \text { for all } u \in W_{0}^{1, \mathcal{H}}(\Omega)
$$

due to the continuity of the embedding from $W_{0}^{1, \mathcal{H}}(\Omega)$ to $L^{r}(\Omega)$ for all $r \in\left(1, p^{*}\right)$. Applying (3.18) and (3.19) in (3.17) and using (3.20) we get

$$
\begin{aligned}
\langle A u & +\xi+\eta-f, u\rangle_{\mathcal{H}} \\
\geq & \|\nabla u\|_{p}^{p}+\|\nabla u\|_{q, \mu}^{q}-\alpha_{j}\|u\|_{\theta}^{\theta}-\left\|\beta_{j}\right\|_{1}-a_{K(w)}\|u\|_{1, \mathcal{H}, 0}-b_{K(w)} \\
& -\|f\|_{W_{0}^{1, \mathcal{H}}(\Omega)^{*}}\|u\|_{1, \mathcal{H}, 0} \\
\geq & \|\nabla u\|_{p}^{p}+\|\nabla u\|_{q, \mu}^{q}-\alpha_{j} c(\theta)^{\theta}\|u\|_{1, \mathcal{H}, 0}^{\theta}-\left\|\beta_{j}\right\|_{1}-a_{K(w)}\|u\|_{1, \mathcal{H}, 0} \\
& -b_{K(w)}-\|f\|_{W_{0}^{1, \mathcal{H}}(\Omega)^{*}}\|u\|_{1, \mathcal{H}, 0} \\
\geq & \min \left\{\|u\|_{1, \mathcal{H}, 0}^{p},\|u\|_{1, \mathcal{H}, 0}^{q}\right\}-\alpha_{j} c(\theta)^{\theta}\|u\|_{1, \mathcal{H}, 0}^{\theta}-\left\|\beta_{j}\right\|_{1} \\
& -a_{K(w)}\|u\|_{1, \mathcal{H}, 0}-b_{K(w)}-\|f\|_{W_{0}^{1, \mathcal{H}}(\Omega)^{*}}\|u\|_{1, \mathcal{H}, 0},
\end{aligned}
$$

where the last inequality is obtained via inequality (2.2). Since $\theta<p<q$, it is clear that we can find a constant $R_{0}>0$ large enough such that $R_{0}^{p}<R_{0}^{q}$ and

$$
R_{0}^{p}-\alpha_{j} c(\theta)^{\theta} R_{0}^{\theta}-\left\|\beta_{j}\right\|_{1}-a_{K(w)} R_{0}-b_{K(w)}-\|f\|_{W_{0}^{1, \mathcal{H}}(\Omega)^{*}} R_{0}>0 .
$$

Therefore, for each $R \geq R_{0}$ fixed, the desired inequality (3.16) holds. 
Suppose now $\theta=p$. Then, taking $W_{0}^{1, \mathcal{H}}(\Omega) \subset W_{0}^{1, p}(\Omega)$ into account and the fact that

$$
\|u\|_{p}^{p} \leq \lambda_{1, p}^{-1}\|\nabla u\|_{p}^{p} \text { for all } u \in W_{0}^{1, p}(\Omega)
$$

we obtain

$$
\begin{aligned}
\langle A u & +\xi+\eta-f, u\rangle_{\mathcal{H}} \\
\geq & \|\nabla u\|_{p}^{p}+\|\nabla u\|_{q, \mu}^{q}-\alpha_{j}\|u\|_{p}^{p}-\left\|\beta_{j}\right\|_{1}-a_{K(w)}\|u\|_{1, \mathcal{H}, 0}-b_{K(w)} \\
& -\|f\|_{W_{0}^{1, \mathcal{H}}(\Omega)^{*}}\|u\|_{1, \mathcal{H}, 0} \\
\geq & \left(1-\alpha_{j} \lambda_{1, p}^{-1}\right)\|\nabla u\|_{p}^{p}+\|\nabla u\|_{q, \mu}^{q}-\left\|\beta_{j}\right\|_{1}-a_{K(w)}\|u\|_{1, \mathcal{H}, 0}-b_{K(w)} \\
- & \|f\|_{W_{0}^{1, \mathcal{H}}(\Omega)^{*}}\|u\|_{1, \mathcal{H}, 0} \\
\geq & \left(1-\alpha_{j} \lambda_{1, p}^{-1}\right)\left(\|\nabla u\|_{p}^{p}+\|\nabla u\|_{q, \mu}^{q}\right)-\left\|\beta_{j}\right\|_{1}-a_{K(w)}\|u\|_{1, \mathcal{H}, 0}-b_{K(w)} \\
& -\|f\|_{W_{0}^{1, \mathcal{H}}(\Omega)^{*}}\|u\|_{1, \mathcal{H}, 0} \\
\geq & \left(1-\alpha_{j} \lambda_{1, p}^{-1}\right) \min \left\{\|u\|_{1, \mathcal{H}, 0}^{p},\|u\|_{1, \mathcal{H}, 0}^{q}\right\}-\left\|\beta_{j}\right\|_{1}-a_{K(w)}\|u\|_{1, \mathcal{H}, 0}-b_{K(w)} \\
& -\|f\|_{W_{0}^{1, \mathcal{H}}(\Omega)^{*}}\|u\|_{1, \mathcal{H}, 0} .
\end{aligned}
$$

Since $1<p<q$ and $\alpha_{j} \lambda_{1, p}^{-1}<1$, we can take $R_{0}>0$ large enough with $R_{0}^{p}<R_{0}^{q}$ such that for all $R \geq R_{0}$ it holds

$$
\left(1-\alpha_{j} \lambda_{1, p}^{-1}\right) R^{p}-\left\|\beta_{j}\right\|_{1}-a_{K(w)} R-b_{K(w)}-\|f\|_{W_{0}^{1, \mathcal{H}}(\Omega)^{*}} R>0 .
$$

Therefore, inequality (3.16) holds and Claim 2 is proved.

Recall that $I_{K(w)}: W_{0}^{1, \mathcal{H}}(\Omega) \rightarrow \overline{\mathbb{R}}$ is a proper, convex and lower semicontinuous function. Hence $\partial_{C} I_{K(w)}: W_{0}^{1, \mathcal{H}}(\Omega) \rightarrow 2^{W_{0}^{1, \mathcal{H}}}(\Omega)^{*}$ is maximal monotone. This fact combined with Claims 1 and 2 allows us to apply Theorem 2.6 which provides $u_{w} \in W_{0}^{1, \mathcal{H}}(\Omega)$ satisfying inclusion (3.9). Thus $\Gamma(w) \neq \emptyset$ for each $w \in W_{0}^{1, \mathcal{H}}(\Omega)$.

Now we want to verify that $\Gamma(w)$ is closed in $W_{0}^{1, \mathcal{H}}(\Omega)$. Let $\left\{u_{n}\right\} \subset \Gamma(w)$ be a sequence such that

$$
u_{n} \rightarrow u \text { in } W_{0}^{1, \mathcal{H}}(\Omega)
$$

for some $u \in W_{0}^{1, \mathcal{H}}(\Omega)$. So, for each $n \in \mathbb{N}$, we have

$$
\left\langle A u_{n}, v-u_{n}\right\rangle_{\mathcal{H}}+J^{\circ}\left(u_{n} ; v-u_{n}\right)+I_{K(w)}(v)-I_{K(w)}\left(u_{n}\right) \geq\left\langle f, v-u_{n}\right\rangle_{\mathcal{H}}
$$

for all $v \in W_{0}^{1, \mathcal{H}}(\Omega)$. Passing to the upper limit as $n \rightarrow \infty$, we get

$$
\begin{aligned}
& \langle A u, v-u\rangle_{\mathcal{H}}+J^{\circ}(u ; v-u)+I_{K(w)}(v)-I_{K(w)}(u) \\
& \quad \geq \limsup _{n \rightarrow \infty}\left[\left\langle A u_{n}, v-u_{n}\right\rangle_{\mathcal{H}}+J^{\circ}\left(u_{n} ; v-u_{n}\right)+I_{K(w)}(v)-I_{K(w)}\left(u_{n}\right)\right] \\
& \quad \geq \limsup _{n \rightarrow \infty}\left\langle f, v-u_{n}\right\rangle_{\mathcal{H}} \\
& \quad=\langle f, v-u\rangle_{\mathcal{H}}
\end{aligned}
$$

for all $v \in W_{0}^{1, \mathcal{H}}(\Omega)$, where we have used the continuity of $A$, see Proposition 2.1, the upper semicontinuity of $(u, v) \mapsto J^{\circ}(u ; v)$, see Proposition 2.4(iv), and the lower semicontinuity of $I_{K(w)}$. This shows that $u \in \Gamma(w)$. Hence, $\Gamma(w)$ is closed. 
Finally, we need to show that $\Gamma(w)$ is bounded. Arguing by contradiction, we suppose that $\Gamma(w)$ is unbounded. Then, there exists a sequence $\left\{u_{n}\right\}$ in $\Gamma(w)$ such that

$$
\left\|u_{n}\right\|_{1, \mathcal{H}, 0} \rightarrow+\infty .
$$

By a simple computing, see (3.21) and (3.23) for example, we are able to find $N_{0} \in \mathbb{N}$ such the for all $n \geq N_{0}$, one has

$$
0 \geq\left\langle A u_{n}, u_{n}\right\rangle_{\mathcal{H}}-J^{\circ}\left(u_{n} ;-u_{n}\right)+I_{K(w)}\left(u_{n}\right)>0,
$$

where we have used the fact that $0 \in K$ and (3.24) which leads to a contradiction. Therefore, $\Gamma(w)$ is bounded.

(ii) Now assume that $\mathrm{H}(f)(\mathrm{v})$ holds. Let $u_{1}, u_{2} \in W_{0}^{1, \mathcal{H}}(\Omega)$ be two solutions of problem (3.7), that is,

$$
\left\langle A u_{i}, v-u_{i}\right\rangle_{\mathcal{H}}+J^{\circ}\left(u_{i} ; v-u_{i}\right)+I_{K(w)}(v)-I_{K(w)}\left(u_{i}\right) \geq\left\langle f, v-u_{i}\right\rangle_{\mathcal{H}}
$$

for all $v \in W_{0}^{1, \mathcal{H}}(\Omega)$ and for $i=1,2$. Taking $v=u_{2}$ and $v=u_{1}$ into the inequalities above for $i=1$ and $i=2$, respectively, and applying (3.4) we obtain

$$
\begin{aligned}
0 \geq & \left\langle A u_{1}-A u_{2}, u_{1}-u_{2}\right\rangle_{\mathcal{H}}-\left(J^{\circ}\left(u_{1} ; u_{2}-u_{1}\right)+J^{\circ}\left(u_{2} ; u_{1}-u_{2}\right)\right) \\
= & \int_{\Omega}\left(\left|\nabla u_{1}\right|^{p-2} \nabla u_{1}-\left|\nabla u_{2}\right|^{p-2} \nabla u_{2}\right) \cdot \nabla\left(u_{1}-u_{2}\right) d x \\
& +\int_{\Omega} \mu(x)\left(\left|\nabla u_{1}\right|^{q-2} \nabla u_{1}-\left|\nabla u_{2}\right|^{q-2} \nabla u_{2}\right) \cdot \nabla\left(u_{1}-u_{2}\right) d x \\
& -m_{j}\left\|u_{1}-u_{2}\right\|_{p}^{p} \\
\geq & \int_{\Omega}\left(\left|\nabla u_{1}\right|^{p-2} \nabla u_{1}-\left|\nabla u_{2}\right|^{p-2} \nabla u_{2}\right) \cdot \nabla\left(u_{1}-u_{2}\right) d x-m_{j}\left\|u_{1}-u_{2}\right\|_{p}^{p},
\end{aligned}
$$

where we have used the fact $\mu(x) \geq 0$ for a. a. $x \in \Omega$. Taking

$$
\int_{\Omega}\left(\left|\nabla u_{1}\right|^{p-2} \nabla u_{1}-\left|\nabla u_{2}\right|^{p-2} \nabla u_{2}\right) \cdot \nabla\left(u_{1}-u_{2}\right) d x \geq c(p)\left\|\nabla\left(u_{1}-u_{2}\right)\right\|_{p}^{p},
$$

into account gives

$$
\begin{aligned}
0 & \geq\left\langle A u_{1}-A u_{2}, u_{1}-u_{2}\right\rangle_{\mathcal{H}}-\left(J^{\circ}\left(u_{1} ; u_{2}-u_{1}\right)+J^{\circ}\left(u_{2} ; u_{1}-u_{2}\right)\right) \\
& \geq c(p)\left\|\nabla\left(u_{1}-u_{2}\right)\right\|_{p}^{p}-m_{j}\left\|u_{1}-u_{2}\right\|_{p}^{p} \\
& \geq\left(c(p)-m_{j} \lambda_{1, p}^{-1}\right)\left\|\nabla\left(u_{1}-u_{2}\right)\right\|_{p}^{p},
\end{aligned}
$$

where the last inequality is obtained by using inequality (3.22). Since $W_{0}^{1, \mathcal{H}}(\Omega) \subset W_{0}^{1, p}(\Omega)$ we deduce that $u_{1}=u_{2}$. Consequently, problem (3.7) has a unique solution in $W_{0}^{1, \mathcal{H}}(\Omega)$.

Now we are in the position to prove Theorem 3.5 by applying Theorem 3.6 and Kluge's fixed point theorem.

Proof of Theorem 3.5 We have already mentioned that the fixed point set of $\Gamma$ is the corresponding set of solutions to problem (3.6). Besides, Lemma 3.2 points out that the set of solutions for problem (3.6) is a subset of the set of solutions for problem (1.1). Consequently, it suffices to show that the set of fixed points of $\Gamma$ is nonempty.

Claim 3 The graph of $\Gamma$ is sequentially weakly closed. 
Let $\left\{w_{n}\right\} \subset W_{0}^{1, \mathcal{H}}(\Omega)$ be a sequence such that $w_{n} \rightarrow w$ and $u_{n}:=\Gamma\left(w_{n}\right) \rightarrow u$ in $W_{0}^{1, \mathcal{H}}(\Omega)$ for some $w, u \in W_{0}^{1, \mathcal{H}}(\Omega)$. Then, for each $n \in \mathbb{N}$ we have $u_{n} \in K\left(w_{n}\right)$, namely $T\left(u_{n}\right) \leq$ $U\left(w_{n}\right)$, and

$$
\left\langle A u_{n}, v-u_{n}\right\rangle_{\mathcal{H}}+J^{\circ}\left(u_{n} ; v-u_{n}\right) \geq\left\langle f, v-u_{n}\right\rangle_{\mathcal{H}}
$$

for all $v \in K\left(w_{n}\right)$. The inequality

$$
\begin{aligned}
& \left\langle A u_{1}-A u_{2}, u_{1}-u_{2}\right\rangle_{\mathcal{H}}-\left(J^{\circ}\left(u_{1} ; u_{2}-u_{1}\right)+J^{\circ}\left(u_{2} ; u_{1}-u_{2}\right)\right) \\
& \geq\left(c(p)-m_{j} \lambda_{1, p}^{-1}\right)\left\|\nabla\left(u_{1}-u_{2}\right)\right\|_{p}^{p} \geq 0
\end{aligned}
$$

for all $u_{1}, u_{2} \in W_{0}^{1, \mathcal{H}}(\Omega)$ and smallness condition (3.5) indicate that $u_{n}$ solves the following problem

$$
\left\langle A v, v-u_{n}\right\rangle_{\mathcal{H}}+J^{\circ}\left(v ; v-u_{n}\right) \geq\left\langle f, v-u_{n}\right\rangle_{\mathcal{H}}
$$

for all $v \in K\left(w_{n}\right)$.

From hypotheses $\mathrm{H}(T)$ and $\mathrm{H}(U)$ we know that

$$
T(u) \leq \limsup _{n \rightarrow \infty} T\left(u_{n}\right) \leq \limsup _{n \rightarrow \infty} U\left(w_{n}\right) \leq U(w) .
$$

This means that $u \in K(w)$.

For any fixed $v \in K(w)$, since $U(w)>0$, we can consider the sequence $\left\{v_{n}\right\}$ defined by

$$
v_{n}:=\frac{U\left(w_{n}\right)}{U(w)} v \text { for all } n \in \mathbb{N} .
$$

The nonnegativity of $U$, the positive homogeneity of $T$ and $v \in K(w)$, that is, $T(v) \leq U(w)$, imply

$$
T\left(v_{n}\right)=T\left(\frac{U\left(w_{n}\right)}{U(w)} v\right)=\frac{U\left(w_{n}\right)}{U(w)} T(v) \leq \frac{U\left(w_{n}\right) U(w)}{U(w)}=U\left(w_{n}\right) .
$$

Hence $v_{n} \in K\left(w_{n}\right)$. A simple calculating gives

$$
\lim _{n \rightarrow \infty}\left\|v_{n}-v\right\|_{1, \mathcal{H}, 0}=\lim _{n \rightarrow \infty}\left|U\left(w_{n}\right)-U(w)\right| \frac{\|v\|_{1, \mathcal{H}, 0}}{U(w)}=0 .
$$

This shows that $v_{n} \rightarrow v$. Taking $v=v_{n}$ in (3.26) and passing to the upper limit as $n \rightarrow \infty$ one has

$$
\begin{aligned}
& \langle A v, v-u\rangle_{\mathcal{H}}+J^{\circ}(v ; v-u) \\
& \quad \geq \limsup _{n \rightarrow \infty}\left\langle A v_{n}, v_{n}-u_{n}\right\rangle_{\mathcal{H}}+\limsup _{n \rightarrow \infty} J^{\circ}\left(v_{n} ; v_{n}-u_{n}\right) \\
& \quad \geq \limsup _{n \rightarrow \infty}\left[\left\langle A v_{n}, v_{n}-u_{n}\right\rangle_{\mathcal{H}}+J^{\circ}\left(v_{n} ; v_{n}-u_{n}\right)\right] \\
& \quad \geq \limsup _{n \rightarrow \infty}\left\langle f, v_{n}-u_{n}\right\rangle_{\mathcal{H}}=\langle f, v-u\rangle_{\mathcal{H}},
\end{aligned}
$$

where we have used the compact embedding of $W_{0}^{1, \mathcal{H}}(\Omega)$ in $L^{q_{1}}(\Omega)$ and the fact that $L^{q_{1}}(\Omega) \times$ $L^{q_{1}}(\Omega) \ni(v, u) \rightarrow J^{\circ}(u ; v) \in \mathbb{R}$ is upper semicontinuous, see (2.3) and Proposition 2.4. Hence,

$$
\langle A v, v-u\rangle_{\mathcal{H}}+J^{\circ}(v ; v-u) \geq\langle f, v-u\rangle_{\mathcal{H}}
$$


for all $v \in K(w)$. Note that $u \in K(w)$ and $K(w)$ is closed and convex. Let $t \in(0,1)$ be arbitrary and set $v_{t}:=t v+(1-t) u$. Taking $v=v_{t}$ in (3.27) and applying Proposition 2.4(i), we get that

$$
\left\langle A v_{t}, v-u\right\rangle_{\mathcal{H}}+J^{\circ}\left(v_{t} ; v-u\right) \geq\langle f, v-u\rangle_{\mathcal{H}} .
$$

Passing to the upper limit in the inequality above as $t \rightarrow 0^{+}$yields

$$
\langle A u, v-u\rangle_{\mathcal{H}}+J^{\circ}(u ; v-u) \geq\langle f, v-u\rangle_{\mathcal{H}}
$$

for all $v \in K(w)$ which means $u=\Gamma(w)$. Therefore, we conclude that the graph of $\Gamma$ is sequentially weakly closed and so Claim 3 is proved.

Claim 4 The set $\Gamma\left(W_{0}^{1, \mathcal{H}}(\Omega)\right)$ is bounded in $W_{0}^{1, \mathcal{H}}(\Omega)$.

Arguing by contradiction and suppose the claim is not true. Then there exists a sequence $\left\{w_{n}\right\}$ such that

$$
\left\|u_{n}\right\|_{1, \mathcal{H}, 0} \rightarrow \infty
$$

where $u_{n}=\Gamma\left(w_{n}\right)$. For every $n \in \mathbb{N}$, one has (3.25) for all $v \in K\left(w_{n}\right)$. Having in mind that $0 \in K(w)$ for each $w \in W_{0}^{1, \mathcal{H}}(\Omega)$, we take $v=0$ as test function in (3.25) to obtain

$$
\left\langle A u_{n}, u_{n}\right\rangle_{\mathcal{H}}-J^{\circ}\left(u_{n} ;-u_{n}\right) \leq\|f\|_{W_{0}^{1, \mathcal{H}}(\Omega)^{*}}\left\|u_{n}\right\|_{1, \mathcal{H}, 0} .
$$

By using the same arguments as in the proof of Theorem 3.6, see (3.21) and (3.23), we can find $N_{0} \in \mathbb{N}$ large enough with $\left\|u_{n}\right\|_{1, \mathcal{H}, 0}^{p}<\left\|u_{n}\right\|_{1, \mathcal{H}, 0}^{q}$ for all $n \geq N_{0}$ such that

$$
0<\left\langle A u_{n}, u_{n}\right\rangle_{\mathcal{H}}-J^{\circ}\left(u_{n} ;-u_{n}\right)-\|f\|_{W_{0}^{1, \mathcal{H}}(\Omega) *}\left\|u_{n}\right\|_{1, \mathcal{H}, 0} \leq 0
$$

for all $n \geq N_{0}$. This is a contradiction. Therefore, we conclude that the set $\Gamma\left(W_{0}^{1, \mathcal{H}}(\Omega)\right)$ is bounded in $W_{0}^{1, \mathcal{H}}(\Omega)$, which proves Claim 4.

Now we can apply Theorem 2.5 for the mapping $\Psi=\Gamma$. This shows that $\Gamma$ admits a fixed point in $W_{0}^{1, \mathcal{H}}(\Omega)$ which implies that problem (1.1) has at least one weak solution in $W_{0}^{1, \mathcal{H}}(\Omega)$. We still need to show that the set $\mathcal{S}$ is bounded and weakly closed.

The boundedness of $\mathcal{S}$ can be obtained directly via using analogous arguments as in the proof of Claim 4.

It remains to show the weak closedness of $\mathcal{S}$. Let $\left\{u_{n}\right\} \subset \mathcal{S}$ be a sequence such that $u_{n} \rightarrow u$ in $W_{0}^{1, \mathcal{H}}(\Omega)$ for some $u \in W_{0}^{1, \mathcal{H}}(\Omega)$. Hence, for each $n \in \mathbb{N}$, we see that $u_{n} \in K\left(u_{n}\right)$ and

$$
\left\langle A v, v-u_{n}\right\rangle_{\mathcal{H}}+\int_{\Omega} j^{\circ}\left(v(x) ; v(x)-u_{n}(x)\right) d x \geq\left\langle f, v-u_{n}\right\rangle_{\mathcal{H}}
$$

for all $v \in K\left(u_{n}\right)$. Because the graph of $K$ is sequentially weakly closed, see Claim 3, this implies $u \in K(u)$. For any $v \in K(u)$, we set $v_{n}:=\frac{U\left(u_{n}\right)}{U(u)} v$. We have $v_{n} \in K\left(u_{n}\right)$ and $v_{n} \rightarrow v$ in $W_{0}^{1, \mathcal{H}}(\Omega)$. Taking $v=v_{n}$ in (3.28) and passing to the upper limit as $n \rightarrow \infty$ we obtain

$$
\langle A v, v-u\rangle_{\mathcal{H}}+\int_{\Omega} j^{\circ}(v(x) ; v(x)-u(x)) d x \geq\langle f, v-u\rangle_{\mathcal{H}}
$$

for all $v \in K(u)$, where we have applied Fatou's Lemma. Invoking the Minty approach gives $u \in \mathcal{S}$. Therefore, $\mathcal{S}$ is weakly closed in $W_{0}^{1, \mathcal{H}}(\Omega)$. 
Acknowledgements Project supported by the H2020-MSCA-RISE-2018 Research and Innovation Staff Exchange Scheme Fellowship within the Project No. 823731 CONMECH, the Startup Project of Doctor Scientific Research of Yulin Normal University No. G2020ZK07, and National Science Center of Poland under Preludium Project No. 2017/25/N/ST1 /00611. It is also supported by the International Project co-financed by the Ministry of Science and Higher Education of Republic of Poland under Grant No. 3792/GGPJ/H2020/2017/0.

Open Access This article is licensed under a Creative Commons Attribution 4.0 International License, which permits use, sharing, adaptation, distribution and reproduction in any medium or format, as long as you give appropriate credit to the original author(s) and the source, provide a link to the Creative Commons licence, and indicate if changes were made. The images or other third party material in this article are included in the article's Creative Commons licence, unless indicated otherwise in a credit line to the material. If material is not included in the article's Creative Commons licence and your intended use is not permitted by statutory regulation or exceeds the permitted use, you will need to obtain permission directly from the copyright holder. To view a copy of this licence, visit http://creativecommons.org/licenses/by/4.0/.

\section{References}

1. Bahrouni, A., Rădulescu, V.D., Repovš, D.D.: Double phase transonic flow problems with variable growth: nonlinear patterns and stationary waves. Nonlinearity 32(7), 2481-2495 (2019)

2. Bahrouni, A., Rădulescu, V.D., Winkert, P.: A critical point theorem for perturbed functionals and low perturbations of differential and nonlocal systems. Adv. Nonlinear Stud. 20(3), 663-674 (2020)

3. Bahrouni, A., Rădulescu, V.D., Winkert, P.: Double phase problems with variable growth and convection for the Baouendi-Grushin operator (2019)

4. Baroni, P., Colombo, M., Mingione, G.: Harnack inequalities for double phase functionals. Nonlinear Anal. 121, 206-222 (2015)

5. Baroni, P., Colombo, M., Mingione, G.: Non-autonomous functionals, borderline cases and related function classes. St. Petersb. Math. J. 27, 347-379 (2016)

6. Baroni, P., Kuusi, T., Mingione, G.: Borderline gradient continuity of minima. J. Fixed Point Theory Appl. 15(2), 537-575 (2014)

7. Baroni, P., Colombo, M., Mingione, G.: Regularity for general functionals with double phase. Calc. Var. Partial Differ. Equ. 57, no. 2, Art. 62 (2018)

8. Brezis, H.: Functional Analysis, Sobolev Spaces and Partial Differential Equations. Springer, New York (2011)

9. Carl, S., Le, V.K., Motreanu, D.: Nonsmooth Variational Problems and Their Inequalities. Springer, New York (2007)

10. Cencelj, M., Rădulescu, V.D., Repovš, D.D.: Double phase problems with variable growth. Nonlinear Anal. 177, 270-287 (2018)

11. Chang, K.C.: Variational methods for nondifferentiable functionals and their applications to partial differential equations. J. Math. Anal. Appl. 80(1), 102-129 (1981)

12. Clarke, F.H.: Optimization and Nonsmooth Analysis. Society for Industrial and Applied Mathematics (SIAM), Philadelphia (1990)

13. Colasuonno, F., Squassina, M.: Eigenvalues for double phase variational integrals. Ann. Mat. Pura Appl. (4) 195(6), 1917-1959 (2016)

14. Colombo, M., Mingione, G.: Bounded minimisers of double phase variational integrals. Arch. Ration. Mech. Anal. 218(1), 219-273 (2015)

15. Colombo, M., Mingione, G.: Regularity for double phase variational problems. Arch. Ration. Mech. Anal. 215(2), 443-496 (2015)

16. Cupini, G., Marcellini, P., Mascolo, E.: Local boundedness of minimizers with limit growth conditions. J. Optim. Theory Appl. 166(1), 1-22 (2015)

17. Gasiński, L., Papageorgiou, N.S.: Constant sign and nodal solutions for superlinear double phase problems. Adv. Calc. Var. https://doi.org/10.1515/acv-2019-0040

18. Gasiński, L., Papageorgiou, N.S.: Nonsmooth Critical Point Theory and Nonlinear Boundary Value Problems. Chapman and Hall/CRC, Boca Raton, FL (2005)

19. Gasiński, L., Papageorgiou, N.S.: Positive solutions for nonlinear elliptic problems with dependence on the gradient. J. Differ. Equ. 263, 1451-1476 (2017)

20. Gasiński, L., Winkert, P.: Constant sign solutions for double phase problems with superlinear nonlinearity. Nonlinear Anal. 195, 111739 (2020) 
21. Gasiński, L., Winkert, P.: Existence and uniqueness results for double phase problems with convection term. J. Differ. Equ. 268(8), 4183-4193 (2020)

22. Kamenskii, M., Obukhovskii, V., Zecca, P.: Condensing Multivalued Maps and Semilinear Differential Inclusions in Banach Spaces. Walter de Gruyter \& Co., Berlin (2001)

23. Kluge, R.: On some parameter determination problems and quasi-variational inequalities. In: Theory of Nonlinear Operators, vol. 6, pp. 129-139. Akademie-Verlag, Berlin (1978)

24. Le, V.K.: A range and existence theorem for pseudomonotone perturbations of maximal monotone operators. Proc. Amer. Math. Soc. 139(5), 1645-1658 (2011)

25. Lê, A.: Eigenvalue problems for the $p$-Laplacian. Nonlinear Anal. 64(5), 1057-1099 (2006)

26. Liu, W., Dai, G.: Existence and multiplicity results for double phase problem. J. Differ. Equ. 265(9), 4311-4334 (2018)

27. Marcellini, P.: The stored-energy for some discontinuous deformations in nonlinear elasticity. In: Partial Differential Equations and the Calculus of Variations, vol. II”, vol. 2, pp. 767-786. Birkhäuser Boston, Boston (1989)

28. Marcellini, P.: Regularity and existence of solutions of elliptic equations with $p, q$-growth conditions. J. Differ. Equ. 90(1), 1-30 (1991)

29. Migórski, S., Ochal, A., Sofonea, M.: Nonlinear Inclusions and Hemivariational Inequalities. Springer, New York (2013)

30. Papageorgiou, N.S., Rădulescu, V.D., Repovš, D.D.: Double-phase problems and a discontinuity property of the spectrum. Proc. Amer. Math. Soc. 147(7), 2899-2910 (2019)

31. Papageorgiou, N.S., Rădulescu, V.D., Repovš, D.D.: Double-phase problems with reaction of arbitrary growth. Z. Angew. Math. Phys. 69, no. 4, Art. 108 (2018)

32. Papageorgiou, N.S., Winkert, P.: Applied Nonlinear Functional Analysis. An Introduction. De Gruyter, Berlin (2018)

33. Perera, K., Squassina, M.: Existence results for double-phase problems via Morse theory. Commun. Contemp. Math. 20, no. 2, 1750023 (2018)

34. Rădulescu, V.D.: Isotropic and anistropic double-phase problems: old and new. Opuscula Math. 39(2), 259-279 (2019)

35. Zhang, Q., Rădulescu, V.D.: Double phase anisotropic variational problems and combined effects of reaction and absorption terms. J. Math. Pures Appl. (9) 118, 159-203 (2018)

36. Zhikov, V.V.: Averaging of functionals of the calculus of variations and elasticity theory. Izv. Akad. Nauk SSSR Ser. Mat. 50(4), 675-710 (1986)

37. Zhikov, V.V.: On Lavrentiev's phenomenon. Russ. J. Math. Phys. 3(2), 249-269 (1995)

38. Zhikov, V.V.: On some variational problems. Russ. J. Math. Phys. 5(1), 105-116 (1997)

39. Zhikov, V.V., Kozlov, S.M., Oleĭnik, O.A.: Homogenization of Differential Operators and Integral Functionals. Springer, Berlin (1994)

Publisher's Note Springer Nature remains neutral with regard to jurisdictional claims in published maps and institutional affiliations. 